\title{
Gaussian Message Passing Based Passive Localization in the Presence of Receiver Detection Failures
}

\author{
Weijie Yuan ${ }^{1,2}$, Qiaolin $\mathrm{Shi}^{1}$, Nan $\mathrm{Wu}^{1}$, Qinghua Guo ${ }^{3}$, Xiaojing Huang ${ }^{2}$ \\ ${ }^{1}$ School of Information and Electronics, Beijing Institute of Technology, Beijing, China \\ ${ }^{2}$ Faculty of Engineering and Information Technology, University of Technology Sydney, NSW, Australia \\ ${ }^{3}$ School of Electrical, Computer and Telecommunications Engineering, The University of Wollongong, NSW, Australia \\ Email: \{wjyuan,qlshi,wunan\}@bit.edu.cn, qguo@uow.edu.au, xiaojing.huang@uts.edu.au
}

\begin{abstract}
This paper considers the issue of passive localization based on time of arrival (TOA) measurement in the presence of receiver detection failures. In passive localization, the signal sent from the transmitter is reflected or relayed by "passive" target and then received at several distributed receivers. The target's position can be determined by collecting range measurements from all receivers. With a linearized model for range measurements, we build a factor graph model and implement Gaussian message passing algorithm to obtain target location and detect link failures. The Cramér-Rao bound (CRB) is also derived to evaluate the performance of the proposed algorithm. Simulation results verify the effectiveness of the proposed factor graph approach.

Index Terms-Passive Localization, Time of Arrival, Factor Graph, Message Passing, Detection Failure, Cramér-Rao bound
\end{abstract}

\section{INTRODUCTION}

For many applications in wireless networks, to obtain the locations of nodes is demanded and therefore has attracted much interest in the recent years. Generally, the locations of nodes are determined based on some nodes with known positions and range measurements. The measurement techniques can be divided into three categories: angle of arrival (AOA), time of arrival (TOA) and received signal strength (RSS). Amongst all techniques, TOA measurement using ultra-wideband (UWB) signaling can provide high-precision distance estimation [1]. In this paper, we mainly focus on the TOA-based methods.

Different from conventional 'active' localization problems, in passive localization, the signal sent from the transmitter is reflected or relayed by the target and then acquired by the receivers, as shown in Fig. 1. The observed range measurements trace out serval ellipses with the target and receivers as foci. The location estimate of the passive target is given by the intersection of those ellipses. Most existing localization methods are designed for active localization. For TOA based passive localization, only a few papers can be found in the literature from the views of inaccurate positions of receivers and synchronous networks [2]-[4].

In practical implantations, the receivers may fail to obtain the range measurements in harsh environmental conditions. Such failures have to be detected in order to ensure the quality

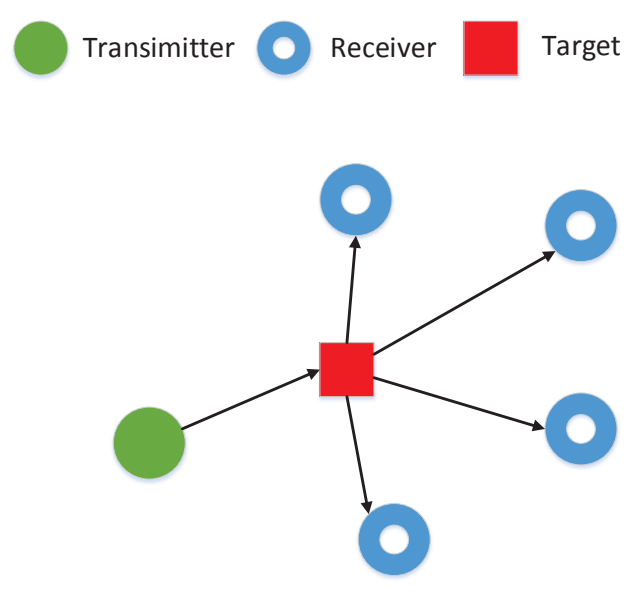

Fig. 1. In passive case, the signal is reflected by the target and acquired at receivers and the location is determined based on the measurements collected at receivers.

of measurements and localization accuracy. For localization, a detection approach based on RSS measurements is proposed in [5], which is effective in indoor environment. Based on graph embeddability and rigidity theory, the authors in [6] build a theoretical foundation to identify detection failures. However, there is little work found in the literature which considers the problem of joint passive localization and link failure detection.

In this paper, we propose a factor graph approach [7] to the joint passive localization and receiver failure detection problem. Based on the factor graph representation, message passing algorithm is employed to determine the "belief" of target's location. Due to the nonlinear Euclidean norm in range measurement, the integration in conventional message updating is intractable. Monte Carlo methods, e.g., particle filtering [8], can solve this problem but with huge complexity. To tackle this problem, we linearize the square root terms in range measurement. Consequently, a linear state space model is derived and Gaussian message passing [9] is employed. Since only multiplication and addition operations are involved in message updating, the computational complexity is reduced 
significantly. Furthermore, the Cramér-Rao bound is derived to evaluate the performance of the proposed algorithms. Simulation results show that the proposed algorithm can improve the performance of passive localization in the presence of detection failures.

Notation: $(\cdot)^{T}$ and $(\cdot)^{-1}$ denote the transpose and the inverse operator, respectively; $\|\cdot\|$ denotes the Euclidean norm; $\hat{r}$ denotes the estimate of an unknown variable; $\mathbb{E}$ denotes the expectation operator; $\delta(\cdot)$ denotes the Dirac delta function; $\propto$ denotes the function on the left hand is proportion to the right hand; the $\nabla_{x}$ denotes the differential operator with respect to $x ; \mathbf{A} \succeq \mathbf{B}$ denotes that $\boldsymbol{A}-\boldsymbol{B}$ is positive semi-definite.

\section{PRELIMINARIES}

Consider a network with one moving target, one static transmitter and $M=|\mathcal{M}|$ fixed receivers, where $\mathcal{M}$ is the set of all receivers, as shown in Fig. 1. Without loss of generality, we denote the location of the target at time $k$ by $\mathbf{x}^{k}=\left[x^{k}, y^{k}\right]^{T}$, the location of transmitter by $\mathbf{x}_{t}=\left[x_{t}, y_{t}\right]^{T}$, the location of the $i$ th receiver $\mathbf{x}_{i}=\left[x_{i}, y_{i}\right]^{T}$. The range measurement from transmitter via the target to the $i$ th receiver is

$$
\bar{z}_{i}^{k}=d_{i}^{k}+n_{i}^{k}=\left\|\mathbf{x}^{k}-\mathbf{x}_{t}\right\|+\left\|\mathbf{x}^{k}-\mathbf{x}_{i}\right\|+n_{i}^{k},
$$

where the noise $n_{i}^{k}$ is typically modeled as Gaussian with zero mean and variance $\sigma_{i}^{2}$. Thus the likelihood function is given by

$p\left(\bar{z}_{i}^{k} \mid \mathbf{x}^{k}\right)=\frac{1}{\sqrt{2 \pi \sigma_{i}^{2}}} \exp \left(-\frac{\left(\bar{z}_{i}^{k}-\left\|\mathbf{x}^{k}-\mathbf{x}_{t}\right\|-\left\|\mathbf{x}^{k}-\mathbf{x}_{i}\right\|\right)^{2}}{2 \sigma_{i}^{2}}\right)$.

If the receiver is working as expected, the observation is the range measurement. In contrast, if the faulty receiver fails to detect the TOA signal, the observation only contains measurement noise, formulated as

$$
z_{i}^{k}=\left\{\begin{array}{cc}
\bar{z}_{i}^{k} & \text { Functioning } \\
n_{i}^{k} & \text { Detection Failure }
\end{array}\right.
$$

\section{PASSIVE Localization USING FACTOR GRAPH APPROACH}

\section{A. Probabilistic Model}

With the prior knowledge of the detection failure occurrence probability $p_{o}$, the likelihood function is given by

$$
\begin{aligned}
p\left(z_{i}^{k} \mid \mathbf{x}^{k}\right) \propto & \left(1-p_{o}\right) \cdot \exp \left(-\frac{\left(z_{i}^{k}-d_{i}^{k}\right)^{2}}{2 \sigma_{i}^{2}}\right) \\
& +p_{o} \cdot \exp \left(-\frac{\left(z_{i}^{k}\right)^{2}}{2 \sigma_{i}^{2}}\right) .
\end{aligned}
$$

We further denote the collection of all range measurements at time $k$ as $\mathbf{z}^{k}=\left[z_{1}^{k}, \ldots, z_{M}^{k}\right]^{T}$ and $\mathbf{z}=\left[\mathbf{z}^{1}, \ldots, \mathbf{z}^{n}\right]$, and the collection of target location to time $n$ as $\mathbf{x}=\left[\mathbf{x}^{1}, \ldots, \mathbf{x}^{n}\right]$. With the assumption that the measurement noise is independent of time stamps and receivers, the global likelihood function is formulated as follows,

$$
p(\mathbf{z} \mid \mathbf{x})=\prod_{k=1}^{n} \prod_{i=1}^{M} p\left(z_{i}^{k} \mid \mathbf{x}^{k}\right) .
$$

The goal is to determine the target location in real time by exploiting all range measurements $\mathbf{z}$ at receivers, equivalently, to determine the posterior distribution of $\mathbf{x}$. Using Bayesian rule,

$$
p(\mathbf{x} \mid \mathbf{z})=p(\mathbf{x}) p(\mathbf{z} \mid \mathbf{x})
$$

where $p(\mathbf{x})$ can be regarded as joint a priori distribution of target location. Since the target is moving continuously, $p(\mathbf{x})$ follows the Markov chain,

$$
p(\mathbf{x})=p\left(\mathbf{x}^{0}\right) \prod_{k=1}^{n} p\left(\mathbf{x}^{k} \mid \mathbf{x}^{k-1}\right)
$$

where $p\left(\mathbf{x}^{k} \mid \mathbf{x}^{k-1}\right)$ is referred to as state transition probability which is related to the state transition function and $p\left(\mathbf{x}^{0}\right)$ is the initial prior information. The state transition function is given as

$$
\mathbf{x}^{k}=\mathbf{x}^{k-1}+\delta_{t} \hat{\mathbf{s}}^{k-1}+\boldsymbol{\Delta}_{s},
$$

where $\hat{\mathbf{s}}^{k-1}=\left[\hat{s}_{x}^{k-1}, \hat{s}_{y}^{k-1}\right]$ is the estimated velocity at time $k-1$, i.e., $\hat{\mathbf{s}}^{k-1}=\left(\hat{\mathbf{x}}^{k-1}-\hat{\mathbf{x}}^{k-2}\right) / c, \delta_{t}$ is duration of the time slot and $\Delta_{s}$ is state transition noise with Gaussian distribution $\mathcal{N}\left(\boldsymbol{\Delta}_{s}, \mathbf{0}, \mathbf{V}_{s}\right)$. Therefore, (6) is factorized as

$$
p(\mathbf{x} \mid \mathbf{z})=p\left(\mathbf{x}^{0}\right) \prod_{k=1}^{n} p\left(\mathbf{x}^{k} \mid \mathbf{x}^{k-1}\right) \prod_{k=1}^{n} \prod_{i=1}^{M} p\left(z_{i}^{k} \mid \mathbf{x}^{k}\right) .
$$

Generally, we focus on the target location at time $k$ under MAP criterion

$$
\hat{\mathbf{x}}^{k}=\arg \max _{\mathbf{x}} p\left(\mathbf{x}^{k} \mid \mathbf{z}\right),
$$

which requires the marginal distribution of $\mathrm{x}^{k}$. Direct marginalization by using $p\left(\mathbf{x}^{k} \mid \mathbf{z}\right)=\sum_{\mathbf{x} \backslash \mathbf{x}^{k}} p(\mathbf{x} \mid \mathbf{z})$ is intractable due to complexity increased exponentially with the scale of $\mathbf{x}$. The factor graph approach is an efficient way to solve this kind of problem by leveraging the conditional independence of variables. However, due to the nonlinear square root terms in range measurement, conventional message passing algorithm [7] is infeasible to implement analytically. To this end, we develop a low complexity algorithm for passive localization based on Gaussian message passing (GMP).

\section{B. The Proposed Algorithm}

Note that the observation $z_{i}^{k}$ in (3) can be written in a general form by introducing a binary state $\psi_{i}^{k}=\{0,1\}$, which is shown as

$$
z_{i}^{k}=\psi_{i}^{k}\left(\left\|\mathbf{x}^{k}-\mathbf{x}_{t}\right\|+\left\|\mathbf{x}^{k}-\mathbf{x}_{i}\right\|\right)+n_{i}^{k},
$$


time $k-1$

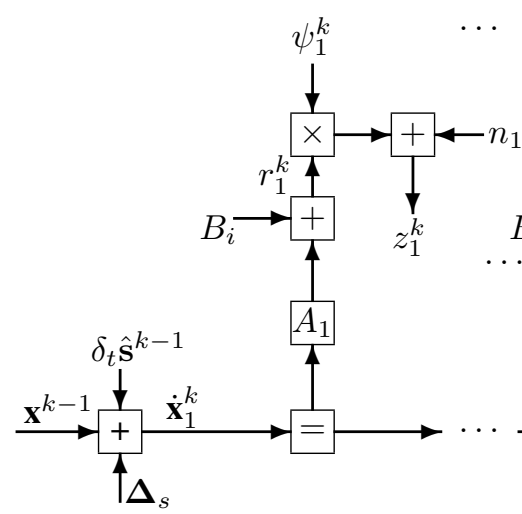

$k$
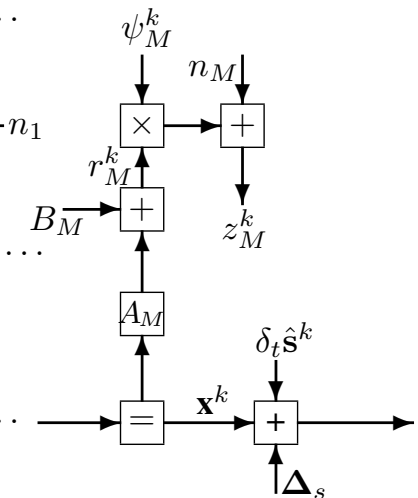

Fig. 2. Factor graph for joint TOA passive localization and receiver failure detection. The node $\times$ denotes the multiplication of two variables, i.e., $z=x \cdot y$.

where $\psi_{i}^{k}=1$ indicates the $i$ th receiver is functioning whereas $\psi_{i}^{k}=0$ indicates the malfunctioning case. The prior distribution of $\psi_{i}^{k}$ is Bernoulli distribution, i.e.,

$$
p\left(\psi_{i}^{k}\right)=p_{o}^{1-\psi_{i}^{k}}\left(1-p_{o}\right)^{\psi_{i}^{k}} .
$$

To tackle the nonlinear square root terms, at time $k$ and in the $l$ th iteration, we expand the Euclidean distance around the previously estimated locations of target by the first order Taylor expansion as,

$$
z_{i}^{k}=\psi_{i}^{k}\left(\mathbf{A}_{i}^{k,(l-1)} \mathbf{x}+B_{i}^{k,(l-1)}\right)+n_{i}^{k},
$$

with $\mathbf{A}_{i}^{k,(l-1)}$ and $B_{i}^{k,(l-1)}$ defined as

$$
\begin{aligned}
\mathbf{A}_{i}^{k,(l-1)}= & \frac{\left(\hat{\mathbf{x}}^{k,(l-1)}-\mathbf{x}_{i}\right)^{T}}{\left\|\hat{\mathbf{x}}^{k,(l-1)}-\mathbf{x}_{i}\right\|}+\frac{\left(\hat{\mathbf{x}}^{k,(l-1)}-\mathbf{x}_{t}\right)^{T}}{\left\|\hat{\mathbf{x}}^{k,(l-1)}-\mathbf{x}_{t}\right\|} \\
B^{k,(l-1)}= & \left\|\hat{\mathbf{x}}^{k,(l-1)}-\mathbf{x}_{i}\right\|+\left\|\hat{\mathbf{x}}^{k,(l-1)}-\mathbf{x}_{t}\right\| \\
& -\mathbf{A}_{i}^{k,(l-1)} \hat{\mathbf{x}}^{k,(l-1)} .
\end{aligned}
$$

Based on the linear state space model given by (8) and (13), the Forney style factor graph (FFG) for joint passive localization and receiver failure detection is depicted in Fig. 2. The Gaussian message passing rules on FFG has been derived in [9]. We use $\mathbf{W}=\mathbf{V}^{-1}$ to denote the weight matrix. Since the product of two unknown variables is involved in the factor graph, we use the multiplier node $\triangle$ to denote the multiplication constraint. In order to apply GMP, we approximate the discrete variable $\psi_{i}^{k}$ as a Gaussian variable by expectation propagation (EP) [10]. Assuming $\overleftarrow{m}_{\psi_{i}^{k}}$ and $\overleftarrow{V}_{\psi_{i}^{k}}$ are available from the previous iteration, the mean and variance of the belief $b\left(\psi_{i}^{k}\right)$ is given as ${ }^{1}$

$$
\begin{aligned}
m_{\psi_{i}^{k}} & =\left(1-p_{o}\right) \frac{1}{\sqrt{2 \pi \overleftarrow{V}_{\psi_{i}^{k}}}} \exp \left(-\frac{\left(1-\overleftarrow{m}_{\psi_{i}^{k}}\right)^{2}}{2 \overleftarrow{V}_{\psi_{i}^{k}}}\right) \\
V_{\psi_{i}^{k}} & =m_{\psi_{i}^{k}}-m_{\psi_{i}^{k}}^{2} .
\end{aligned}
$$

${ }^{1}$ It should be noted that the first and second order moments of $b\left(\psi_{i}^{k}\right)$ are identical.
Then $\vec{m}_{\psi_{i}^{k}}$ and $\vec{V}_{\psi_{i}^{k}}$ are given as

$$
\begin{aligned}
\vec{V}_{\psi_{i}^{k}} & =\frac{V_{\psi_{i}^{k}} \overleftarrow{V}_{\psi_{i}^{k}}}{V_{\psi_{i}^{k}}-\overleftarrow{V}_{\psi_{i}^{k}}}, \\
\vec{m}_{\psi_{i}^{k}} & =\frac{m_{\psi_{i}^{k}} V_{\psi_{i}^{k}}-\overleftarrow{m}_{\psi_{i}^{k}} \overleftarrow{V}_{\psi_{i}^{k}}}{V_{\psi_{i}^{k}}-\overleftarrow{V}_{\psi_{i}^{k}}}
\end{aligned}
$$

Note that for a multiplier node $z=x y$, even the messages $\vec{\mu}_{x}$ and $\overleftarrow{\mu}_{z}$ are Gaussian, the message $\vec{\mu}_{y}$ cannot be written in Gaussian form. To tackle this problem, variational message passing is utilized instead of SPA on the multiplier node. Thereby the corresponding messages are obtained in Gaussian closed form without any further approximation. The derivations of Gaussian VMP are given in Appendix A. The Gaussian messages for the multiplier node are given by

$$
\begin{aligned}
& \overleftarrow{V}_{\psi_{i}^{k}}=\frac{\sigma_{i}^{2}}{V_{r_{i}^{k}}+m_{r_{i}^{k}}^{2}}, \\
& \overleftarrow{m}_{\psi_{i}^{k}}=\frac{z_{i}^{k} m_{r_{i}^{k}}}{V_{r_{i}^{k}}+m_{r_{i}^{k}}^{2}}, \\
& \overleftarrow{V}_{r_{i}^{k}}=\frac{\sigma_{i}^{2}}{V_{\psi_{i}^{k}}+m_{\psi_{i}^{k}}^{2}}, \\
& \overleftarrow{m}_{r_{i}^{k}}=\frac{z_{i}^{k} m_{\psi_{i}^{k}}}{V_{\psi_{i}^{k}}+m_{\psi_{i}^{k}}^{2}},
\end{aligned}
$$

with

$$
\begin{gathered}
V_{r_{i}^{k}}=\overleftarrow{V}_{r_{i}^{k}}+\vec{V}_{r_{i}^{k}}, \\
m_{r_{i}^{k}}=V_{r_{i}^{k}}^{-1}\left(\overleftarrow{W}_{r_{i}^{k}} \overleftarrow{m}_{r_{i}^{k}}+\vec{W}_{r_{i}^{k}} \vec{m}_{r_{i}^{k}}\right) .
\end{gathered}
$$

Then following GMP rules, the belief of target is obtained as

$$
\begin{aligned}
& \overrightarrow{\mathbf{V}}_{\mathbf{x}^{k}}=\left(\overrightarrow{\mathbf{W}}_{\dot{\mathbf{x}}_{1}^{k}}+\sum_{i=1}^{M} \overleftarrow{\mathbf{W}}_{\mathbf{x}_{i}^{k}}\right)^{-1}=\left(\overrightarrow{\mathbf{W}}_{\dot{\mathbf{x}}_{1}^{k}}+\sum_{i=1}^{M} \frac{\mathbf{A}_{i}^{k, T} \mathbf{A}_{i}^{k}}{\overleftarrow{V}_{r_{i}^{k}}}\right)^{-1} \\
& \overrightarrow{\mathbf{m}}_{\mathbf{x}^{k}}=\overrightarrow{\mathbf{V}}_{\mathbf{x}^{k}}\left(\overrightarrow{\mathbf{W}}_{\dot{\mathbf{x}}^{k}} \overrightarrow{\mathbf{m}}_{\dot{\mathbf{x}}^{k}}+\sum_{i=1}^{M} \frac{\mathbf{A}_{i}^{k, T} \overleftarrow{m}_{r_{i}^{k}}}{\overleftarrow{V}_{r_{i}^{k}}}\right)
\end{aligned}
$$

Moreover, after determining $\overleftarrow{m}_{\psi_{i}^{k}}$ and $\overleftarrow{V}_{\psi_{i}^{k}}$, the state of $\psi_{i}^{k}$ can be decided based on log-likelihood ratio (LLR),

$$
\operatorname{LLR}\left(\psi_{i}^{k}\right)=\frac{1-2 \overleftarrow{m}_{\psi_{i}^{k}}}{\overleftarrow{V}_{\psi_{i}^{k}}}+\ln \frac{1-p_{o}}{p_{o}}
$$

If $\operatorname{LLR}\left(\psi_{i}^{k}\right)<0$, we say that the receiver detection fails and vice versa. Finally, it is able to estimate the target location via (26) (27) and decide the states of sensors based on LLR in (28).

Overall, since all messages in the proposed algorithm are described by means and covariance matrices, the computational complexity is much lower than the particle filtering based conventional message passing algorithm. 


\section{CRAmÉR-RAo LOWer BOUnd}

The Cramér-Rao bound (CRB) establishes the lower bound of the variance of an estimator. For parameters $\tau$ to be estimated, it follows the CRB theorem that

$$
\operatorname{cov}(\hat{\boldsymbol{\tau}}) \succeq \mathbf{F}^{-1},
$$

where $\mathbf{F}$ is the Fisher information matrix (FIM). ${ }^{2} \mathbf{F}$ is given by

$$
\begin{aligned}
\mathbf{F} & =-\mathbb{E}\left[\nabla_{\boldsymbol{\tau}}\left\{\nabla_{\boldsymbol{\tau}}\left(\ln p\left(\boldsymbol{\tau} \mid \mathbf{z}^{k}\right)\right)\right\}\right] \\
& =\underbrace{-\mathbb{E}\left[\nabla_{\boldsymbol{\tau}}\left\{\nabla_{\boldsymbol{\tau}}\left(\ln p\left(\mathbf{z}^{k} \mid \boldsymbol{\tau}\right)\right)\right\}\right]}_{\mathbf{F}_{1}}-\underbrace{\mathbb{E}\left[\nabla_{\boldsymbol{\tau}}\left\{\nabla_{\boldsymbol{\tau}}(\ln p(\boldsymbol{\tau}))\right\}\right]}_{\mathbf{F}_{\mathrm{p}}},
\end{aligned}
$$

$\mathbf{F}_{1}$ and $\mathbf{F}_{\mathrm{p}}$ are the Fisher "information" from observations at time $k$ and from previous state. For a vector $\boldsymbol{\tau}=\left[\tau_{1}, \ldots, \tau_{N}\right]^{T}$ of $N$ parameters to be estimated, $\mathbf{F}_{\mathrm{p}}$ is an $N \times N$ diagonal matrix

$$
\mathbf{F}_{\mathrm{p}}=\operatorname{diag}\left\{1 / \sigma_{\tau_{1}}^{2}, \ldots, 1 / \sigma_{\tau_{N}}^{2}\right\}
$$

which is related to the variance of previous time slot and the state transition noise. $\mathbf{F}_{1}$ is also an $N \times N$ matrix with respect to the likelihood function. Next, we will consider the computation of $\mathbf{F}_{1}$ in detail.

For the considered case, $\boldsymbol{\tau}=\mathbf{x}=[x, y]^{T}$. Note that the probability of detection failure occurrence $p_{o}$ may change, thus the CRB is different with respect to different $p_{o}$. Based on the independent assumption of measurement noise, we have

$$
\mathbf{F}_{1}=\mathbb{E}\left[\frac{\partial^{2} \ln \prod_{i} p\left(z_{i} \mid \mathbf{x}\right)}{\partial \mathbf{x}^{2}}\right]=\sum_{i=1}^{M} \mathbb{E}\left[\frac{\partial^{2} \ln p\left(z_{i} \mid \mathbf{x}\right)}{\partial \mathbf{x}^{2}}\right] .
$$

Considering the calculation of $\frac{\partial^{2} \ln p\left(z_{i} \mid \mathbf{x}\right)}{\partial \mathbf{x}^{2}}$, with (4) and the shorthand notation $f\left(z_{i}\right)=-\frac{\left(z_{i}-d_{i}\right)^{2}}{\sigma_{i}^{2}}, f\left(n_{i}\right)=-\frac{z_{i}^{2}}{\sigma_{i}^{2}}$ and const $=-\ln \sqrt{2 \pi \sigma_{i}^{2}}, \ln p\left(z_{i} \mid \mathbf{x}\right)$ follows

$$
\begin{gathered}
\ln p\left(z_{i} \mid \mathbf{x}\right)=\text { const }+\ln \left(\left(1-p_{o}\right) e^{f\left(z_{i}\right)}+p_{o} e^{f\left(n_{i}\right)}\right), \\
=\mathrm{const}+\ln \left(e^{f\left(z_{i}\right)+\ln \left(1-p_{o}\right)}+e^{f\left(n_{i}\right)+\ln p_{o}}\right) .
\end{gathered}
$$

Based on (33), the FIM $\mathbf{F}_{1}$ can not be given analytically but can only be determined by means of numerical method. In order to derive an analytical FIM, we further employ the Jacobian logarithm to simplify (33). It is well known that, for real numbers $a_{1}$ and $a_{2}$

$$
\ln \left(e^{a_{1}}+e^{a_{2}}\right)=\max \left\{a_{1}, a_{2}\right\}+\ln \left(1+e^{-\left|a_{1}-a_{2}\right|}\right) .
$$

Generally, for large $\left|a_{1}-a_{2}\right|, \ln \left(1+e^{-\left|a_{1}-a_{2}\right|}\right) \approx 0$. Based on this approximation, (33) can be approximated as

$$
\begin{gathered}
\ln p\left(z_{i} \mid \mathbf{x}\right) \approx \operatorname{const}+\max \left\{f\left(z_{i}\right)+\ln \left(1-p_{o}\right), f\left(n_{i}\right)+\ln p_{o}\right\} \\
= \begin{cases}\ln \frac{1-p_{o}}{\sqrt{2 \pi \sigma_{i}^{2}}}-\frac{\left(z_{i}-d_{i}\right)^{2}}{\sigma_{i}^{2}}, & d_{i}\left(2 z_{i}-d_{i}\right) \geq \epsilon \\
\ln \frac{p_{o}}{\sqrt{2 \pi \sigma_{i}^{2}}}-\frac{\left(z_{i}\right)^{2}}{\sigma_{i}^{2}}, & d_{i}\left(2 z_{i}-d_{i}\right)<\epsilon\end{cases}
\end{gathered}
$$

\footnotetext{
${ }^{2}$ Since the localization system is time-variant, here we analyze the CRB at time $k$ for example. The superscript $k$ is omitted for simplicity.
}

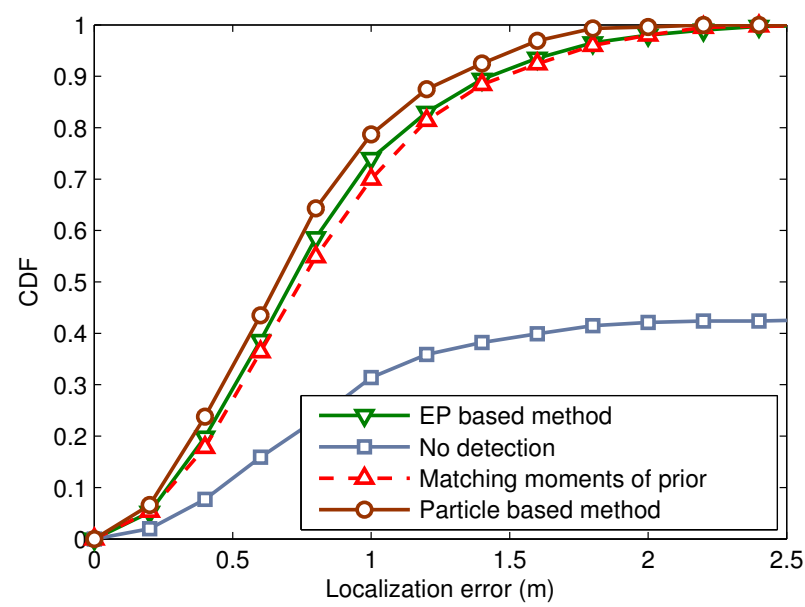

Fig. 3. CDF of target location of proposed algorithm with the occupance probability $p_{o}=0.2$

where the threshold $\epsilon=\sigma_{i}^{2} \ln \frac{p_{o}}{1-p_{o}}$.

For the case $\ln p\left(z_{i} \mid \mathbf{x}\right) \approx \ln \frac{1-p_{o}}{\sqrt{2 \pi \sigma_{i}^{2}}}-\frac{\left(z_{i}-d_{i}\right)^{2}}{\sigma_{i}^{2}}$, the second order partial derivative has the form

$$
\frac{\partial^{2} \ln p\left(z_{i} \mid \mathbf{x}\right)}{\partial \mathbf{x}^{2}}=\frac{1}{\sigma_{i}^{2}}\left[\begin{array}{cc}
J_{11}^{2} & J_{11} J_{21} \\
J_{21} J_{11} & J_{21}^{2}
\end{array}\right]
$$

where $J_{11}=\frac{x-x_{t}}{\sqrt{\left(x-x_{t}\right)^{2}+\left(y-y_{t}\right)^{2}}}+\frac{x-x_{1}}{\sqrt{\left(x-x_{1}\right)^{2}+\left(y-y_{1}\right)^{2}}}$ and $J_{21}=\frac{y-y_{t}}{\sqrt{\left(x-x_{t}\right)^{2}+\left(y-y_{t}\right)^{2}}}+\frac{y-y_{1}}{\sqrt{\left(x-x_{1}\right)^{2}+\left(y-y_{1}\right)^{2}}}$. For the other case, the partial derivative results in a zero matrix 0 which means in this case, the measurement cannot provide any "information" for locating the target. The total FIM F can then be given based on (32), and the CRB is given by $\operatorname{cov}(\hat{\mathbf{x}}) \succeq \mathbf{F}^{-1}$.

\section{Simulation Results and Discussions}

In this section, we evaluate the performance of proposed algorithms. A $200 \times 200 \mathrm{~m}^{2}$ plane with one moving target, one transmitter and 6 receivers is considered in the simulations. The transceivers are assumed to be located at fixed positions, i.e., $\mathbf{x}_{t}=[0,0]^{\mathrm{T}}, \mathbf{x}_{1}=[80,10]^{\mathrm{T}}, \mathbf{x}_{2}=[10,80]^{\mathrm{T}}$, $\mathbf{x}_{3}=[130,20]^{\mathrm{T}}, \mathbf{x}_{4}=[20,130]^{\mathrm{T}}, \mathbf{x}_{5}=[180,30]^{\mathrm{T}}$ and $\mathbf{x}_{6}=[30,180]^{\mathrm{T}}$. For brevity, the measurement noise is set to $\sigma_{i}^{2}=2 \mathrm{~m}^{2}, \forall i \in \mathcal{M}$ unless specified. We consider 20 time slots and the number of message passing iterations at a single time slot is $L=10$.

In Fig. 3, the CDF of target location with the detection failure occupance probability $p_{o}=0.2$ is plotted. Obviously, when the faulty measurements observed at sensors are used without detection, the localization performance is seriously affected. The particle based message passing without Gaussian approximation has the best performance at the cost of huge complexity. We can further observe that using EP to approximate the discrete variable to Gaussian has superior performance than matching the moments of its prior directly. This is because EP can exploit the information obtained from 


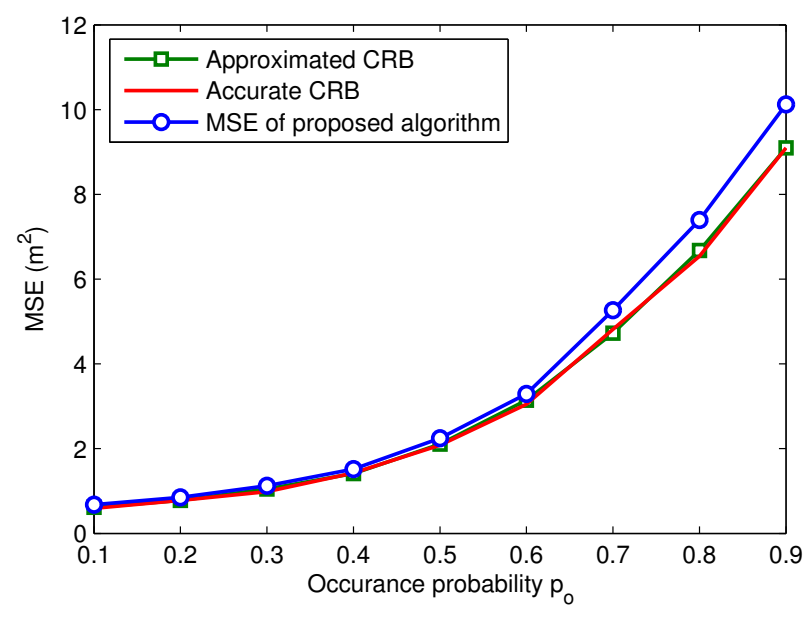

Fig. 4. MSE of proposed algorithm and the derived CRB versus the occupance probability $p_{o}$

the measurements, or in other words, the extrinsic information. Then in Fig. 4 the mean squared error (MSE) of target location with varying $p_{o} \in[0.1,0.9]$ is depicted. The approximated CRB derived from the FIM (36) and true CRB obtained using numerical method are drawn as benchmarks. It is observed that the approximated CRB is close to the true one. In the region of small $p_{o}$, the proposed is shown to be robustness to the detection failures. When $p_{o}$ becomes larger, the MSE of the proposed algorithm and its CRB both degrade due to insufficient number of functioning receivers. In this situation, the Fisher information $\mathbf{F}$ solely depends on $\mathbf{F}_{b}$ from previous state.

\section{CONCLUSIONS}

In this paper, we consider the issue of receiver detection failures in TOA based passive localization based on a factor graph framework and employ Gaussian message passing techniques to solve it efficiently. To reduce the complexity of particle based method, we linearized the range measurement to obtain a linear state-space model for the proposed passive localization problem. Then the Cramér-Rao bound is derived to evaluate the localization performance. Simulation results show that the performance of proposed algorithm is close to that of the high complexity particle based method and can attain the Cramér-Rao bound.

\section{APPENDIX A \\ DERIVATION OF GAUSSIAN VARIATIONAL MESSAGE PASSING}

Consider a multiplication constraint $\delta(z-x y)$, under the SPA rules and Gaussian assumption of $\mu_{y \rightarrow \delta}(y) \propto$ $\mathcal{N}\left(y, m_{y}, V_{y}\right)$, the message $\mu_{\delta \rightarrow x}$ is given as

$$
\begin{aligned}
\mu_{\delta \rightarrow x}(x) & =\int \delta(z-x y) \mu_{y \rightarrow \delta}(y) \mathrm{d} y \\
& =\exp \left(-\frac{\left(\frac{z}{x}-m_{y}\right)^{2}}{2 V_{y}}\right)=\exp \left(-\frac{m_{y}^{2}\left(x-\frac{z}{m_{y}}\right)^{2}}{2 x^{2} V_{y}}\right) .
\end{aligned}
$$

Note that the equation (A1) can not be written in Gaussian since variable $x$ is in both the numerator and denominator. Correspondingly, the message $\mu_{\delta \rightarrow y}(y)$ suffers the same problem. In some communication problem like "turbo" equalization, the variable $x$ may hold the energy normalization constraint that $x^{2}=1$, then $\mu_{\delta \rightarrow x}(x)$ is still Gaussian. However, this constraint is not available in the localization problem since the range measurement can not be normalized. Therefore to implement the low complexity Gaussian message passing, we employ the variational message passing (VMP) [11].

The message in VMP is obtained via expectation of the log likelihood function with respect to other variables, i.e.

$$
\mu_{f \rightarrow x}(x)=\exp \left(\mathbb{E}_{\mathbf{y}}(\ln f(x, \mathbf{y}) b(\mathbf{y}))\right)
$$

where $b(\mathbf{y})$ is the belief of $\mathbf{y}$. Considering the multiplier node in Fig. 2, the observation model is $z_{i}^{k}=\phi_{i}^{k} r_{i}^{k}+n_{i}$. Hence the message from $凶$ to $\phi_{i}^{k}$ is calculated as,

$$
\begin{aligned}
& \mu_{\times \rightarrow \phi_{i}^{k}}\left(\phi_{i}^{k}\right) \propto \\
& C \cdot \exp \left(-\int \frac{\left(z_{i}^{k}-\phi_{i}^{k} r_{i}^{k}\right)^{2}}{2 \sigma_{i}^{2}} \exp \left(-\frac{\left(r_{i}^{k}-m_{r_{i}^{k}}\right)^{2}}{2 V_{r_{i}^{k}}}\right)\right) \\
& \propto \exp \left(-\left(\phi_{i}^{k}\right)^{2} \frac{V_{r_{i}^{k}}+m_{r_{i}^{k}}^{2}}{\sigma^{2}}+2 \phi_{i}^{k} \frac{z_{i}^{k} m_{r_{i}^{k}}}{\sigma^{2}}\right) \\
& \propto \mathcal{N}\left(\phi_{i}^{k}, \overleftarrow{m}_{\phi_{i}^{k}}, \overleftarrow{V}_{\phi_{i}^{k}}\right),
\end{aligned}
$$

with $\overleftarrow{m}_{\phi_{i}^{k}}$ and $\overleftarrow{V}_{\phi_{i}^{k}}$ shown in (20) and (21). $\overleftarrow{m}_{r_{i}^{k}}$ and $\overleftarrow{V}_{r_{i}^{k}}$ are determined in a similar way, which is also in Gaussian form.

\section{REFERENCES}

[1] E. Xu, Z. Ding, and S. Dasgupta, "Source Localization in Wireless Sensor Networks From Signal Time-of-Arrival Measurements," IEEE Trans. Signal Process., vol. 59, pp. 2887-2897, 2011.

[2] N. Wu, W. Yuan, H. Wang, and J. Kuang, "Toa-based passive localization of multiple targets with inaccurate receivers based on belief propagation on factor graph," Digital Signal Process., vol. 49, pp. 14-23, 2016.

[3] Y. Wang, S. Ma, and C. Chen, "TOA-based passive localization in quasisynchronous networks," IEEE Commun. Lett., vol. 18, no. 4, pp. 592595, April 2014.

[4] J. Shen, A. F. Molisch, and J. Salmi, "Accurate passive location estimation using TOA measurements," IEEE Trans. Wireless Commun., vol. 11, no. 6, pp. 2182-2192, 2012.

[5] Y. C. Chen and J. C. Juang, "Outlier-detection-based indoor localization system for wireless sensor networks," Int. J. Nav. Obser., vol. 2012, 2012.

[6] L. Jian, Z. Yang, and Y. Liu, "Beyond triangle inequality: Sifting noisy and outlier distance measurements for localization," in Proc. 2010 IEEE INFOCOM, March 2010, pp. 1-9.

[7] F. R. Kschischang, B. J. Frey, and H.-A. Loeliger, "Factor graphs and the sum-product algorithm," IEEE Trans. Inf. Theory, vol. 47, no. 2, pp. 498-519, 2001.

[8] P. M. Djurić, J. H. Kotecha, J. Zhang, Y. Huang, T. Ghirmai, M. F. Bugallo, and J. Miguez, "Particle filtering," IEEE Signal Process. Mag., vol. 20, no. 5, pp. 19-38, 2003.

[9] H.-A. Loeliger, J. Dauwels, J. Hu, S. Korl, L. Ping, and F. R. Kschischang, "The factor graph approach to model-based signal processing," Proc. IEEE, vol. 95, no. 6, pp. 1295-1322, 2007.

[10] T. P. Minka, "Expectation propagation for approximate bayesian inference," in Proc. Conf. Uncertainty Artif. Intell. Morgan Kaufmann Publishers Inc., 2001, pp. 362-369.

[11] J. M. Winn and C. M. Bishop, "Variational message passing," in $J$. Mach. Learn. Res., 2005, pp. 661-694. 